\title{
Heart-Water : essai de traitement par l'auréomycine
}

\author{
par H. CASSARD
}

En 1955 et en 1956, plusieurs cas de Heart-Water surviennent dans une exploitation des environs de Brazzaville sur du bétail Friesland importé d'Afrique du Sud. Après le premier ces sur lequel il ne put être posé de diagnostic certain, nous avons essaye un traitement par l'auréomycine à la dose de $5 \mathrm{mg}$ par $\mathrm{kg}$ de poids vif par voie intraveineuse. Parmi une dizaine d'animaux présentant une forme aiguë ou suraiguë et que nous avons immédiatement traités, aucun cas de guérison n'a pu être enregistré. Nous avons parfois réussi à prolonger les malades de quelques jours mais tous sont morts dans un laps de temps allant de quelques heures à une semaine. A l'autopsie, tous ces animaux possédaient des lésions de Heart-Water et des rickettsies du type Rickettsia Ruminantium furent mises en évidence dans les cellules endothéliales des capillaires sur frottis de substance nerveuse prélevee dans la corne d'Ammon (*).

En juin 1957, à la " Station Administrative du kilomètre dix-sept " près de Brazzaville, une dizaine de jeunes bovins dont l'âge variait de 10 à 15 mois présentèrent un état d'amaigrissement extrême avec diarrhée profuse ne cédant pas aux diverses médications classiques instituées. Trois de ces animaux moururent au pâturage sans que nous ayons pu en pratiquer l'autopsie. Quelques jours plus tard, deux taurillons moururent le même jour et l'autopsie faite révéla les lésions suivantes considérées comme caractéristiques de la Heart-Water dans ses formes à evolution lente:

Etat cachectique.

Muqueuses légèrement congestionnées. Muqueuse nasale présentant quelques pétechies avec mucus mêlé de filets de sang.

(*) Les examens des preparations microscopiques et les dêterminations ont été faits par le Docteur J. Oro à l'Institut Pasteur de Brazzavilue.
Inflammation catarrhale de l'intestin grêle et plus particulièrement du duodénum. Présence de larges plaques rouges congestives sur la muqueuse de la caillette. Aucune lésion particulière sur le foie mais légère distension de la vésicule biliaire.

Aucune lésion pulmonaire. Nombreuses pétéchies à la surface du cœur formant un pointillé plus ou moins dense le long du sillon auriculoventriculaire et dans les sillons longitudinaux, le long des vaisseaux coronaires. Pas d'exsudat péricardique.

Légère congestion cérébrale.

Des frottis de corne d'Ammon faits aussitôt après la mort révèlèrent la présence de Rickettsia Ruminantium dans les cellules endothéliales des capillaires.

A titre expérimental, nous décidons de prendre deux des animaux parmi les malades restants et de tenter un traitement à l'auréomycine à forte dose. Les deux animaux traités reçoivent, l'un 13 milligrammes, l'autre 20 milligrammes d'auréomycine par kilogramme de poids vif et par jour administrés par voie veineuse.

Le premier meurt après 4 jours de traitement. L'autopsie pratiquée montre les mêmes lésions que sur les animaux morts sans avoir été traités. Ces lésions sont cependant moins nettes. Nous trouvons des rickettsies très nombreuses dans les cellules endothéliales de la corne d'Ammon.

Le second meurt après 5 jours de traitement. Les lésions sont discrètes mais les frottis cffoctués riches en rickettsies.

\section{CONCLUSION}

Il est communément admis que la Heart-Water évoluant sous la forme aiguë peut être combattue efficacement par un traitement à l'auréomycine a 
Condition que celui-ci soit institué très précocement. Nos propres observations ne confirment pas cette efficacité.

Il semble d'autre part que l'auréomycine administrée même à forte dose (10 et $20 \mathrm{mg} / \mathrm{kg}$ ) n'apporte aucune amélioration aux cas chroniques de HeartWater et les rickettsies trouvées dans les cellules endothéliales de la corne d'Ammon sont aussi nombreuses sur les animaux traités que sur les non traités.

\section{SUMMARY}

Trials of the treatment of Heart-Water with aureomycin.

Recovery from an acute form of Heart-Water was not obtained with $5 \mathrm{mg} / \mathrm{kg}$ aureomycin in 10 cattle. Higher dosages - $10-20 \mathrm{mg} / \mathrm{kg}$ - did not improvechronic cases since at post-mortem examination rickettsia were found in the endothelium of cornu Ammonis in similar numbers as in untreated animals.

\section{RESUMEN}

\section{Heart-water : ensayo de tratamiento con Aureomicina.}

El autor habiendo tratado con aureomicina a la dosis de $5 \mathrm{mg} / \mathrm{kg}$ a doce bovinos que presentaban una forma aguda de heart-water no obtuvo jamas curación. Por otra parte un tratamiento con aureomicina a grandes dosis, 10 a $20 \mathrm{mg} / \mathrm{kg}$ no le ha permitido obtener mejoría en las formas crónicas de heart-water. Cada vez, en la autopsia encuentra riketsias tan numerosas en las celulas endoteliales del cuerno de Ammon como en las celulas de animales no tratados. 